\title{
Anderson Localization and Superconductivity
}

\author{
L. N. Bulaevskii \\ P. N. Lebedev Physical Institute, USSR Academy of Sciences, Moscow, USSR
}

\begin{abstract}
M. V. Sadovskii
Institute for Metal Physics, Ural Scientific Centre, USSR Academy of Sciences, Sverdlovsk, USSR
\end{abstract}

(Received July 3, 1984; revised October 11, 1984)

The possibility of superconductivity is considered for a strongly disordered metal approaching the Anderson transition. A microscopic derivation of the coefficients of the Ginzburg-Landau expansion is given for a system in the vicinity of the mobility edge. The localization transition is described within the framework of the self-consistent theory of localization. The superconducting response persists in the localization region. The appropriate change in the behavior of the upper critical field $\mathrm{H}_{\mathrm{c} 2}$ is considered for the localization region. The Coulomb repulsion grows as the Fermi level approaches the mobility edge, leading to a degradation of the superconducting $\mathrm{T}_{\mathrm{c}}$. However, under rather rigid conditions superconductivity is possible both at the mobility edge and in a narrow region below the mobility edge, i.e., in an Anderson insulator. Finally, experimental data for superconducting molybdenum sulfides irradiated by fast neutrons are discussed.

\section{INTRODUCTION}

The concept of localization forms the basis of the modern theory of electrons in strongly disordered systems. ${ }^{1,2}$ Sufficiently strong disorder introduced into an ideal metallic system leads to the localization of electronic states in the vicinity of the Fermi level (Anderson transition). ${ }^{3}$ The electronic density of states at the Fermi level remains finite, but because of spatial localization of the electronic wave functions, dc electrical conductivity at zero temperature is impossible, i.e., the system becomes an insulator. At the same time if there exists an attraction of electrons in the vicinity of the Fermi level, the metallic system becomes superconducting at low temperatures. ${ }^{4}$ So the problem arises of the interplay of these two types of transitions, leading to essentially different ground states of the system (insulator versus superconductor). This question is also important from an 
experimental point of view due to a strong dependence of the superconducting properties of some compounds on the degree of the structural disorder, which can be changed greatly by fast neutron irradiation.

The influence of localization on superconductivity has been dealt with in a number of recent theoretical papers. ${ }^{5-13}$ Attention was paid particularly to the study of localization corrections in two-dimensional superconductors. ${ }^{6-10}$ However, the possibility of superconductivity in the vicinity of a real Anderson transition was not studied. In this paper we address the problem of superconductivity in a three-dimensional metal undergoing an Anderson transition. From the experimental point of view we consider a rather exotic situation. In fact in most metals the Anderson transition is not realized even for the fully amorphous state. This is due to the rather high values of the typical Fermi energy $E_{\mathrm{F}}$. Possible candidates are metals with low values of $E_{\mathrm{F}}$ (semimetals, narrow-band conductors) and also quasi-one-dimensional and quasi-two-dimensional conductors.

In the first part of the paper we treat the problem in the framework of the BCS model, ${ }^{4}$ which assumes the existence of an attractive interaction between electrons near the Fermi level. For this model a statement can be proved (Anderson theorem) ${ }^{14}$ that claims the unimportant influence of structural disorder on the superconducting transition temperature $T_{c}$. The arguments used in this proof are, in fact, independent of whether the electronic states are localized or not. ${ }^{6,8,11}$ However a question arises about the physical meaning of $T_{c}$ in the localization region, as to whether below this temperature the system still has the Meissner response to an external magnetic field and can sustain a persistent current. This problem can be solved by the derivation of the Ginzburg-Landau (GL) equations for the system in the vicinity of the Anderson transition. We shall demonstrate that superconductivity persists for $T<T_{c}$, i.e., an Anderson insulator-superconductor transition is possible. On the basis of the GL equations, we study the behavior of the upper critical field $H_{c 2}$ in the region of the Anderson transition.

To justify the applicability of the BCS model we must show that the electron-phonon mechanism of electron-electron attraction may dominate over Coulomb repulsion even in the localization region. In a recent paper Anderson et al. ${ }^{12}$ demonstrate that the diffusive nature of electron motion in a disordered system leads to the growth of an effective repulsion of electrons forming Cooper pairs and to the appropriate suppression of $T_{c}$ with disorder. We shall show, however, that under rather rigid conditions the value of $T_{c}$ remains finite both at the mobility edge and in some narrow region below the mobility edge (Anderson insulator), although it is the growth of the Coulomb repulsion that leads to the destruction of superconductivity in the insulator phase at some critical disorder. 
Finally, we discuss some experiments on superconductors irradiated by fast neutrons, which give some evidence on the possible realization of our theoretical estimates in real systems.

\section{GINZBURG-LANDAU EQUATIONS}

\subsection{General Relations}

Consider the electrons in a disordered system, assuming the existence of an effective electron-electron attraction $g$, in an energy region of the order of $2 \omega_{\mathrm{D}}$ around the Fermi level ( $\omega_{\mathrm{D}}$ is the Debye frequency). To study the problem of superconductivity in such a system we must not only discuss the value of $T_{c}$, but also consider the response to an external vector potential A.

In the general case, the study of response functions for a superconducting system with localized one-electron states presents a rather difficult problem. However, near $T_{c}$ the problem simplifies, and in fact we must only show that the free energy density for our system can be represented by the standard GL form ${ }^{15,16}$

$$
F=F_{n}+A|\Delta|^{2}+\frac{1}{2} B|\Delta|^{4}+C\left|\left(\frac{\partial}{\partial \mathbf{r}}-\frac{2 i e}{\hbar c} \mathbf{A}\right) \Delta\right|^{2}
$$

where $F_{n}$ is the free energy density for the normal state and $\Delta$ is the superconducting order parameter. Now the problem reduces to the microscopic derivation of the coefficients $A, B$, and $C$ in (1), taking into account the possibility of electron localization in the disordered system, thus generalizing the results of Gorkov"15,16 for "dirty" superconductors. In the following we use the system of units $\hbar=1$, restoring the value of $\hbar$ only in some final expressions.

Within the BCS model the coefficients $A$ and $B$ in fact do not change in comparison with the ordinary theory of "dirty" superconductors, even as we approach the mobility edge, so long as the Anderson theorem can be applied. Below we shall determine the appropriate conditions. Less trivial is the behavior of the coefficients $C$, which in fact determines the superconducting response. In the limit of ordinary "dirty" superconductors it is proportional to the diffusion coefficient of electrons, i.e., to the conductivity at $T=0$. As we approach the mobility edge this conductivity goes to zero. However, we shall show that in the region of the Anderson transition $C$ remains finite even in the region of localized states.

To determine the coefficients of the GL expansion it is sufficient to study the two-particle Green's functions for the normal system. ${ }^{15} \mathrm{We}$ introduce two-particle Matsubara Green's functions for electrons in the normal 
system in the momentum representation ${ }^{17-19}$ :

$$
\begin{aligned}
\psi\left(\mathbf{q} \omega_{m} \varepsilon_{n}\right)= & -\frac{1}{2 \pi i} \int \frac{d^{3} \mathbf{p}}{(2 \pi)^{3}} \int \frac{d^{3} \mathbf{p}^{\prime}}{(2 \pi)^{3}} \\
& \times\left\langle G\left(\mathbf{p}_{+} \mathbf{p}_{+}^{\prime}-\varepsilon_{n}+\omega_{m}\right) G\left(-\mathbf{p}_{-}^{\prime}-\mathbf{p}_{-}-\varepsilon_{n}\right)\right\rangle \\
\phi\left(\mathbf{q} \omega_{m} \varepsilon_{n}\right)= & -\frac{1}{2 \pi i} \int \frac{d^{3} \mathbf{p}}{(2 \pi)^{3}} \int \frac{d^{3} \mathbf{p}^{\prime}}{(2 \pi)^{3}} \\
& \times\left\langle G\left(\mathbf{p}_{+} \mathbf{p}_{+}^{\prime}-\varepsilon_{n}+\omega_{m}\right) G\left(\mathbf{p}_{-}^{\prime} \mathbf{p}_{-}-\varepsilon_{n}\right)\right\rangle
\end{aligned}
$$

where the angular brackets denote averaging over the random configurations of the disordered system, $\mathbf{p}_{ \pm}=\mathbf{p} \pm \frac{1}{2} \mathbf{q}, \varepsilon_{n}=(2 n+1) \pi T$, and $\omega_{m}=2 \pi m T$. Graphically these functions are represented (for $\omega_{m}=2 \varepsilon_{n}$ ) in Fig. 1, where shaded blocks denote the exact vertex parts in the standard impurity diagram technique.

Then for the coefficients $A, B$, and $C$ we get ${ }^{9,15,16}$

$$
\begin{aligned}
& A=\frac{1}{g}+2 \pi i T \sum_{\varepsilon_{n}} \psi\left(\mathbf{q}=0, \omega_{m}=2 \varepsilon_{n}\right) \\
& C=\left.i \pi T \sum_{\varepsilon_{n}} \frac{\partial^{2}}{\partial q^{2}} \psi\left(\mathbf{q} \omega_{m}=2 \varepsilon_{n}\right)\right|_{q=0}
\end{aligned}
$$

We see that the superconducting properties are determined by the function $\psi$ describing the propagation of two electrons. At the same time the function $\phi$ describes the kinetic properties of the normal state and the localization transition. In the case of time-reversal invariance, i.e., in the absence of external magnetic field and magnetic impurities, we have $\mathrm{e}^{18,19}$

$$
\psi\left(\mathbf{q} \omega_{m}\right)=\phi\left(\mathbf{q} \omega_{m}\right)
$$

and our problem reduces to the calculation of $\phi\left(\mathbf{q} \omega_{m}\right)$.

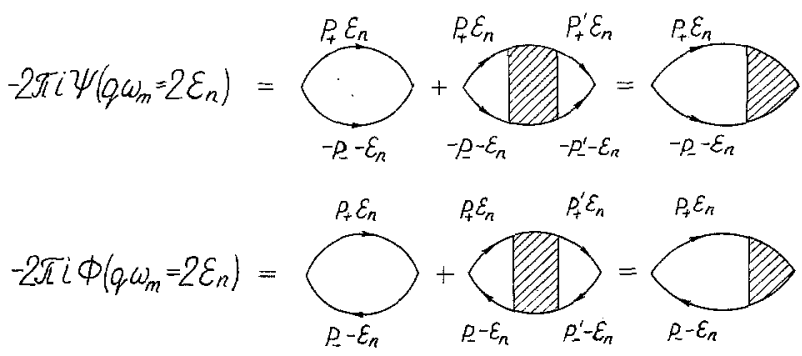

Fig. 1. Diagrammatic representation for $\psi$ and $\phi$. Shaded blocks denote the exact vertexes of the impurity diagram technique. There is no summation over $\varepsilon_{n}$ in the loops. 


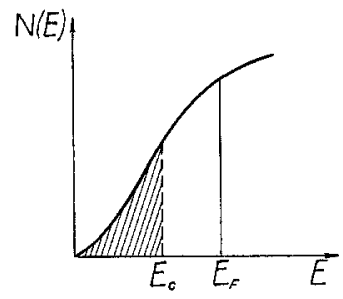

(a)

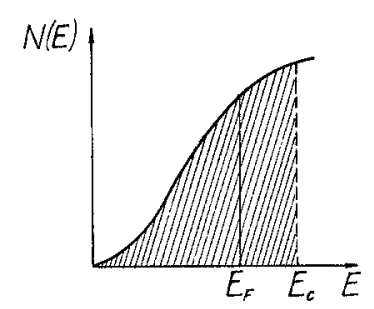

(b)

Fig. 2. Anderson transition showing the density of states in the conduction band. States with $E<E_{c}$ are localized. (a) Metallic phase $\left(E_{\mathrm{F}}>E_{\mathrm{c}}\right) ;\left(\right.$ b) insulator phase $\left(E_{\mathrm{F}}<E_{c}\right)$.

For a disordered system the electronic states of the conduction band are localized near the band edge up to an energy $E_{c}$ (the mobility edge). As the disorder grows, the value of $E_{c}$ moves upward and can pass the Fermi level $E_{\mathrm{F}}$ (see Fig. 2). Thus we have an Anderson transition. As a one-electron model of this transition we take Götze's self-consistent theory of localization in the form proposed by Vollhardt and Wölfe. ${ }^{17-21}$ The main attraction of this theory is the practical possibility of performing calculations for the whole range of parameters of the system, from "good" metal to Anderson insulator. For small $\mathbf{q}$ and $\omega_{m}$ we have

$$
\phi\left(\mathbf{q} \omega_{m}\right)=-\frac{N\left(E_{\mathrm{F}}\right)}{i\left|\omega_{m}\right|+i \tilde{D}\left(\left|\omega_{m}\right|\right) q^{2}}
$$

where the generalized diffusion coefficient at the Fermi level $\tilde{D}\left(\omega_{m}\right)$ is determined by the self-consistency equation ${ }^{17,18}$

$$
\frac{D_{0}}{\tilde{D}\left(\omega_{m}\right)}=1-\frac{i}{\pi N^{2}\left(E_{\mathrm{F}}\right)} \int \frac{d^{3} \mathbf{q}}{(2 \pi)^{3}} \phi\left(\mathbf{q} \omega_{m}\right)
$$

Here $N\left(E_{\mathrm{F}}\right)$ is the electronic density of states at the Fermi level in a disordered system, $D_{0}=\frac{1}{3} v_{\mathrm{F}}^{2} \tau$ is the "bare" diffusion coefficient, $\tau$ is the mean free time in the Born approximation, and $v_{\mathrm{F}}$ is the Fermi velocity. For a model of pointlike random scatterers with scattering amplitude $V$ and spatial density $\rho$ we have $1 / \tau=2 \pi \rho V^{2} N\left(E_{\mathrm{F}}\right)$. In the following the "bare" mean free time $\tau$ and the appropriate mean free path $l=v_{\mathrm{F}} \tau$ will characterize the degree of disorder. In the localization region these parameters obviously do not have the same simple meaning, which is clear in the metallic state.

For the three-dimensional case (7) reduces to ${ }^{18,21}$

$$
\frac{\tilde{D}\left(\omega_{m}\right)}{D_{0}}=1-\frac{\lambda}{\lambda_{c}}-\frac{\pi}{2} \frac{\lambda}{\lambda_{c}}\left[\frac{D_{0}}{\tilde{D}\left(\omega_{m}\right)} \omega_{m} \tau\right]^{1 / 2}
$$


where $\lambda=\left(2 \pi E_{\mathrm{F}} \tau\right)^{-1}$ and $\lambda_{c}$ is the value of $\lambda$ for $E_{\mathrm{F}}=E_{c}$. The solution of (8) can be written approximately as

$$
\tilde{D}\left(\omega_{m}\right) \approx \operatorname{Max}\left[D \frac{\omega_{m}}{\omega_{m}+3 D \omega_{0}^{2} / v_{\mathrm{F}}^{2}} ; D_{0}\left(\omega_{m} \tau\right)^{1 / 3}\right]
$$

where

$$
D=D_{0}\left(k_{\mathrm{F}} R_{l}\right)^{-1}
$$

is the renormalized diffusion coefficient, while the characteristic frequency $\omega_{0}$ is

$$
\omega_{0}^{2}=\left\{\begin{array}{lll}
\frac{1}{3} v_{\mathrm{F}}^{2} R_{I}^{-2} ; & E_{\mathrm{F}}<E_{c} & \text { (insulator) } \\
0 ; & E_{\mathrm{F}} \geqslant E_{c} & \text { (metal) }
\end{array}\right.
$$

Here $R_{l}$ is the correlation length for the Anderson transition. ${ }^{1,18,20}$ For $E_{\mathrm{F}}$ near $E_{c}$ we have

$$
R_{l}=\frac{1}{k_{\mathrm{F}}}\left|1-\frac{\lambda}{\lambda_{c}}\right|^{-\nu} \approx \frac{1}{k_{\mathrm{F}}}\left|\frac{E_{\mathrm{F}}-E_{c}}{E_{c}}\right|^{-\nu}
$$

where $\nu$ is the critical exponent. In the self-consistent theory, for the three-dimensional case $\nu=1$; however, experimentally the value may differ. The frequency $\omega_{0}$ is in many respects similar to an order parameter in the usual theory of phase transitions. It becomes nonzero in the localized phase and determines the insulator nature of the electromagnetic response, e.g., the dielectric function. ${ }^{17-20,31}$ In principle it is a measurable characteristic of the localized phase and gives information on $R_{l}$ [see (11)] in the insulator region in the same manner as $\sigma$ defines it in the metal region (see below).

The position of the mobility edge in the conduction band for free electrons in the model of pointlike scatterers is determined by the estimate ${ }^{20}$

$$
E_{c}=\frac{9}{2 \pi^{4}} m^{3}\left(\rho V^{2}\right)^{2}=\left.\frac{9}{4 \pi^{2}} E_{\mathrm{F}}\left(E_{\mathrm{F}} \tau\right)^{-2}\right|_{E_{\mathrm{F}}=E_{c}}
$$

At the mobility edge $\left(E_{\mathrm{F}}=E_{c}\right)$ we have $E_{\mathrm{F}} \tau=3 / 2 \pi$ or $k_{\mathrm{F}} l=3 / \pi$. With the growth of disorder, i.e., of the value of $\rho V^{2}, \tau$ diminishes and $R_{l}$ grows in the metallic region $\left(E_{\mathrm{F}}>E_{\mathrm{c}}\right)$, while the renormalized diffusion coefficient (and conductivity) drops to zero at the transition at $E_{\mathrm{F}}=E_{c}$, where $R_{l}=\infty$. With further growth of disorder we enter the localization region, where $R_{l}$ determines the localization length of an electron. Here $R_{l}$ drops as $E_{\mathrm{F}}$ moves deep into the localization region, while $\omega_{0}$ grows, similarly to the growth of an order parameter in the condensed phase in the theory of phase transitions. 
Note that the equations of the self-consistent theory of localization are derived with the essential use of perturbation theory over the parameter $\left(E_{\mathrm{F}} \tau\right)^{-1}$. Actually, as we have seen above, this parameter is not small at the mobility edge, and the self-consistent theory in fact has no controllable small parameter. ${ }^{20}$

For the metallic phase $\left(E_{\mathrm{F}} \geqslant E_{c}\right)$ the experimentally measurable static conductivity $\sigma$ is determined by the renormalized diffusion coefficient and can be expressed as

$$
\sigma=2 e^{2} D N\left(E_{\mathrm{F}}\right)=\sigma_{0} / k_{\mathrm{F}} R_{l}=\sigma_{0}-\sigma_{c}
$$

where $\sigma_{0}=2 e^{2} D_{0} N\left(E_{\mathrm{F}}\right)$ is the usual Drude conductivity and $\sigma_{c}$ is its value at the mobility edge $\left(E_{\mathrm{F}}=E_{c}\right): \sigma_{c}=e^{2} k_{\mathrm{F}} / \pi^{3} \hbar$. The last equality in (14) is valid for $\nu=1$. We shall use this relation following from the self-consistent theory to simplify the analysis, although, as we have already stressed, the experimental value of $\nu$ may be quite different and for $\sigma_{0} \approx \sigma_{c}$ the relation (14) is replaced by

$$
\sigma \approx \sigma_{c}\left(\frac{\sigma_{0}-\sigma_{c}}{\sigma_{c}}\right)^{\nu}
$$

In most experiments on the strongly disordered metals the typical scale for $\sigma_{0}$ is determined by the Ioffe-Regel limit ${ }^{22}$; the mean free path is of the order of a few interatomic distances and $\sigma \approx \sigma_{0} \approx 10^{3} \Omega^{-1} \mathrm{~cm}^{-1}$. However, near the Anderson transition the value of $\sigma_{0}$ drops to $\sigma_{c}$, which is obviously of the order of the minimum metallic conductivity due to Mott and Davis ${ }^{23}$ : $\sigma_{c} \approx(2-5) \times 10^{2} \Omega^{-1} \mathrm{~cm}^{-1}$. This is a characteristic conductivity scale for the continuous Anderson transition. ${ }^{1}$ Using (14), we can, in principle, relate $\sigma_{0}$ to the experimental value of the conductivity $\sigma: \sigma_{0}=\sigma_{c}+\sigma$. However, the value of $\sigma_{c}$ should be considered as an obvious fitting parameter, to be determined from experimental dependences.

An obvious limitation of our theory is the explicit neglect of the effect of the electron-electron interaction upon the metal-insulator transition in the disordered system. We assume the validity of the picture of the Anderson transition $^{3}$ as described in the one-electron approximation. However, it is known $\mathrm{n}^{31,32}$ that the electron interaction has an important effect in the vicinity of this transition. Within the framework of the BSC model where the only interelectron interaction is an attraction in the vicinity of the Fermi level,

\footnotetext{
*The Ioffe-Regel conductivity region is characterized by a very low negative temperature coefficient of resistivity and strictly speaking cannot be described by the usual BoltzmannDrude theory. In most of this paper we are concerned with still lower values of the conductivity, of the order of $\sigma_{c}$ typical of the vicinity of the Anderson transition. However, it is possible that the actual behavior of the system in the Ioffe-Regel region is intimately connected to localization.
} 
described by the pairing constant $g$, we are free to assume the weakness of this interaction, i.e., $g N\left(E_{\mathrm{F}}\right) \ll 1$, and neglect its influence upon the pair propagator $\phi\left(q, \omega_{m}\right)$ completely. In a real system we apparently have to consider electron-phonon and Coulomb interactions and disorder on an equal footing (see below). Unfortunately, there is no complete theory of the metal-insulator transition in disordered systems.

Thus we limit ourselves to the study of the coexistence of localization and superconductivity in the framework of the BCS model in the weak coupling limit.

\subsection{Coefficients of the GL Expansion}

The details of the calculations leading to the final expressions for the GL coefficients $A, B$, and $C$ are given in Appendix A. Here we quote only the results. The coefficients $A$ and $B$, determining the transition temperature and the order parameter near $T_{c}$, in complete accordance with the Anderson theorem, are described by the usual expressions for "dirty" superconductors ${ }^{15,16}$ :

$$
\begin{aligned}
& A=N\left(E_{\mathrm{F}}\right) \ln \frac{T}{T_{c}} \approx N\left(E_{\mathrm{F}}\right) \frac{T-T_{c}}{T_{c}} \\
& B=\frac{7 \zeta(3)}{8 \pi^{2} T_{c}^{2}} N\left(E_{\mathrm{F}}\right) ; \quad T_{c}=1.13 \omega_{\mathrm{D}} e^{-1 / \lambda}
\end{aligned}
$$

where $\lambda=g N\left(E_{\mathrm{F}}\right)$. These expressions depend on disorder only through $N\left(E_{\mathrm{F}}\right)$, but they are valid even below the mobility edge $\left(E_{\mathrm{F}}<E_{c}\right)$, i.e., in an Anderson insulator.

Significant changes occur in the coefficient $C$ of the gradient term of the GL expansion. Using (4)-(6) and (9), we find

$$
C \approx\left\{\begin{array}{l}
\frac{\pi}{8 T_{c}} N\left(E_{\mathrm{F}}\right) D ; \quad R_{l}<\left(\xi_{0} l^{2}\right)^{1 / 3} ; E_{\mathrm{F}}>E_{c} \\
N\left(E_{\mathrm{F}}\right)\left(\frac{D_{0} l}{T_{c}}\right)^{2 / 3} \approx N\left(E_{\mathrm{F}}\right)\left(\xi_{0} l^{2}\right)^{2 / 3} ; \quad R_{l}>\left(\xi_{0} l^{2}\right)^{1 / 3} ; E_{\mathrm{F}} \gtrless E_{c} \\
N\left(E_{\mathrm{F}}\right) R_{l}^{2} \ln \frac{1.78 D}{\pi T_{c} R_{l}^{2}} ; \quad R_{l}<\left(\xi_{0} l^{2}\right)^{1 / 3} ; E_{\mathrm{F}}<E_{c}
\end{array}\right.
$$

where $\xi_{0}=0.18 v_{\mathrm{F}} / T_{c}$ is the superconducting coherence length. In the metallic region, as the Fermi energy $E_{\mathrm{F}}$ approaches the mobility edge $E_{c}$, the characteristic length $R_{I}$ grows and the coefficient $C$ diminishes as the renormalized diffusion coefficient $D$ from (10) and is proportional to the metallic conductivity (14). However, in the vicinity of the mobility edge, 
as $\sigma \rightarrow 0, C$ diminishes more slowly and remains finite even for $E_{\mathrm{F}}<E_{c}$ (Anderson insulator). With further lowering of $E_{\mathrm{F}}$ deep into the localization region, $C$ is determined by the localization length $R_{l}$, which diminishes as $E_{\mathrm{F}}$ moves apart from $E_{c}$.

Our analysis of the insulator region $\left(E_{\mathrm{F}}<E_{c}\right)$ is limited to the range of sufficiently large $R_{l}$, such that ${ }^{5}$

$$
\left[N\left(E_{\mathrm{F}}\right) R_{l}^{3}\right]^{-1} \ll T_{c}
$$

This is the condition of a large number of discrete energy levels within a sphere of radius $R_{l}$ in the energy interval $T_{c}$, which is the necessary condition for Cooper pairing of localized electrons. It is easy to see that (17) reduces to

$$
R_{l} \gg\left[N\left(E_{\mathrm{F}}\right) T_{c}\right]^{-1 / 3} \sim\left(\xi_{0} / k_{\mathrm{F}}^{2}\right)^{1 / 3} \sim\left(\xi_{0} l^{2}\right)^{1 / 3} ; \quad E_{\mathrm{F}}<E_{c}
$$

Thus the final asymptotics in (16) in fact has no region of applicability, and within the BCS model the condition of superconductivity in the insulator phase is given by (17) and (18). The meaning of these results is that the electron motion within a localization region of size $R_{l}$ is sufficient to produce coherent Cooper pairs.

For the superconducting correlation length $\xi(T)$ and the London penetration length $\lambda_{\mathrm{L}}$ we obtain, using (1) and (14)-(16) (cf. Ref. 16)

$$
\begin{gathered}
\xi^{2}(T) \approx\left(\frac{T_{c}-T}{T_{c}}\right)^{-1} \begin{cases}\xi_{0} l \frac{\sigma}{\sigma+\sigma_{c}} ; & \sigma>\sigma^{*}\left(E_{\mathrm{F}}>E_{c}\right) \\
\left(\xi_{0} l^{2}\right)^{2 / 3} ; & \sigma<\sigma^{*}\left(E_{\mathrm{F}} \gtrless E_{c}\right)\end{cases} \\
\lambda_{\mathrm{L}}^{-2}=32 \pi e^{2} c^{-2} N\left(E_{\mathrm{F}}\right) \Delta^{2} \xi^{2}(T)\left(1-T / T_{c}\right)
\end{gathered}
$$

From (19) we can see that both $\xi^{2}(T)$ and $\lambda_{\mathrm{L}}^{-2}$ initially drop proportionally to $\sigma$, while the disorder grows, but already in the metallic region, for $R_{l} \geqslant\left(\xi_{0} l^{2}\right)^{1 / 3}$, these quantities diminish more slowly than the conductivity. This change of behavior starts for

$$
\sigma \leqslant \sigma^{*} \approx \sigma_{c}\left(k_{\mathrm{F}} \xi_{0}\right)^{-1 / 3}
$$

which is the key quantity for the effect of localization on the superconducting coherence length. For typical values of $\xi_{0} \sim 100 l$ and $l \sim k_{\mathrm{F}}^{-1}$ we have $\sigma^{*} \leqslant 10^{2} \Omega^{-1} \mathrm{~cm}^{-1}$, i.e., the value of $\sigma^{*}$ is smaller in general than the minimal metallic conductivity. However, it is again better to understand it as a parameter to be determined from experiments, showing the transition to a behavior different from the predictions of the usual theory of "dirty" superconductors.

For the insulator phase the values of $\xi^{2}(T)$ and $\lambda_{L}^{-2}$ remain finite, although they diminish further with $l$. The critical current for a thin supercon- 
ducting plate is proportional to $\lambda_{\mathrm{L}}^{-2} / \xi(T),{ }^{16}$ and remains finite after $\sigma$ becomes zero.

Finally, we note that our results give evidence of localization destruction for electrons forming Cooper pairs. However, the character of the wave functions and kinetic properties for one-particle excitations below $T_{c}$ are at present unknown.

\subsection{The Upper Critical Field}

Direct information about $\xi^{2}(T)$ can be obtained through measurements of the upper critical field $H_{c 2},{ }^{16}$

$$
H_{c 2}=\phi_{0} / 2 \pi \xi^{2}(T) ; \quad \phi_{0}=\pi c \hbar / e
$$

Using (19), we obtain a relation connecting $\sigma,\left(d H_{c 2} / d T\right)_{T_{c}}$, and the value of $N\left(E_{\mathrm{F}}\right)$, which can be determined through independent measurements of the electronic specific heat:

$$
-\frac{\sigma}{N\left(E_{\mathrm{F}}\right)}\left(\frac{d H_{c 2}}{d T}\right)_{T_{c}} \approx \begin{cases}\frac{8 e^{2}}{\pi^{2} \hbar} \phi_{0} ; \quad \sigma>\sigma^{*} & \\ \frac{\phi_{0}}{2 \pi} \frac{\sigma}{\left[N\left(E_{\mathrm{F}}\right) T_{c}\right]^{1 / 3}} ; & \sigma<\sigma^{*}\end{cases}
$$

On the rhs of (22a) only fundamental constants appear, and this relation is often used for the interpretation of measurements on irradiated superconductors. ${ }^{24,25}$ Using this relation, we can find values of $N\left(E_{\mathrm{F}}\right)$ for different degrees of disorder from the measured values of $\left(d H_{c 2} / d T\right)_{T_{c}}$ and conductivity $\sigma$. However, near the metal-insulator transition, when $\sigma \leqslant \sigma^{*}$, this relation is already invalid and the described method of interpretation of measurements of $\left(d H_{c 2} / d T\right)_{T_{c}}$ simulates a fall of $N\left(E_{\mathrm{F}}\right)$ with $\sigma$ according to (22b). In real systems this behavior was observed in Refs. 24 and 25 and we stress the importance of independent experiments to determine $N\left(E_{\mathrm{F}}\right)$. According to preliminary data obtained by the authors of Ref. 24 via specific heat measurements, the value of $N\left(E_{\mathrm{F}}\right)$ remains practically unchanged with the growth of disorder.

Here it is appropriate to note that our derivation of the coefficient $C$ essentially used time-reversal invariance, as expressed by (5), which is correct in the absence of external magnetic field (and magnetic impurities). So our results are formally correct in the limit of an infinitesimal external field, which is sufficient for the demonstration of the superconducting (Meissner) response and for the determination of $\left(d H_{c 2} / d T\right)_{T_{c}}$, because $H_{c 2} \rightarrow 0$ for $T \rightarrow T_{c}$ (see below). In a finite external field we must take into account the influence of the magnetic field upon localization, which is 
expressed by the violation of (5). This problem is far from being solved. However, if we neglect this influence, we can calculate the full dependence of the orbital upper critical field $H_{c 2}(T)$. This is determined by the equation ${ }^{26}$

$$
\ln \frac{T}{T_{c}}=\pi T \sum_{\varepsilon_{n}}\left\{\frac{1}{\left|\varepsilon_{n}\right|+\tilde{D}\left(2\left|\varepsilon_{n}\right|\right) \pi H / \phi_{0}}-\frac{1}{\left|\varepsilon_{n}\right|}\right\}
$$

where $\tilde{D}\left(2 \varepsilon_{n}\right)$ is determined from Eq. (8). Introducing the parameter

$$
a=1.23 \frac{\sigma}{\sigma^{*}}\left[1+\frac{\sigma}{\sigma^{*}}\left(k_{\mathrm{F}} \xi_{0}\right)^{-1 / 3}\right]^{-1}
$$

and calculating $b_{n}, x$, and $S$ via the equations

$$
\begin{gathered}
2 n+1=b_{n}\left(b_{n}-a\right)^{2} ; \quad b_{n}>a ; \quad S=\sum_{n=0}^{\infty} b_{n}(2 n+1)^{-2} \\
{\left[1+\frac{x}{2(1+x)}\right] \ln x+\frac{\pi}{2} \frac{\sqrt{x}}{1+x}+\ln 3.56 a^{3}=0}
\end{gathered}
$$

we obtain the characteristic parameters:

$$
\begin{gathered}
r(a)=-\frac{H_{c 2}(0)}{T_{c}\left(d H_{c 2} / d T\right)_{T_{c}}}=2 a^{2} x S \\
k(a)=-\frac{\pi^{2} \sigma}{8 N\left(E_{\mathrm{F}}\right) e^{2} \phi^{0}}\left(\frac{d H_{c 2}}{d T}\right)_{T_{c}}=\frac{\pi^{2} a}{8 S}
\end{gathered}
$$

These dependences are represented in Fig. 3. As we approach the mobility edge, $r(a)$ grows from the standard value of 0.69 typical for "dirty" superconductors ${ }^{26}$ to the value 1.24 in the localization region, i.e., for $\sigma \ll \sigma^{*}$. This growth of $r(a)$ transforms the positive curvature in the dependence of

$$
h_{c 2}(T)=-H_{c 2}(T) / T_{c}\left(d H_{c 2} / d T\right)_{T_{c}}
$$

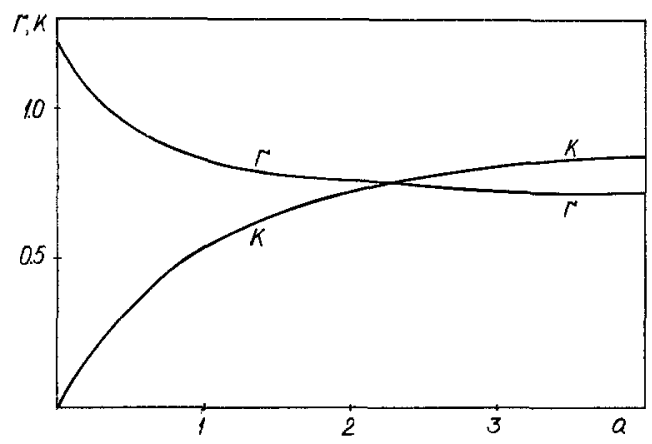

Fig. 3. Calculated dependences of $r$ and $k$ of Eqs. (25) on the effective disorder parameter $a$ [Eq. (24a)]. 


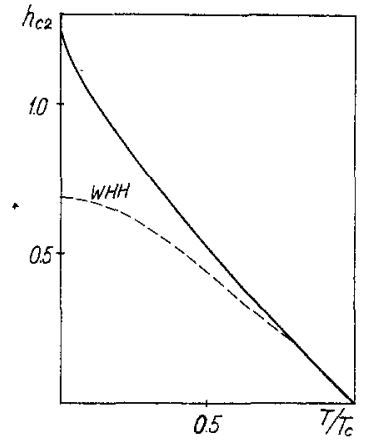

Fig. 4. Calculated dependence of $h_{c 2}(T)=-H_{\mathrm{c} 2}(T) / T_{c}\left(d H_{c 2} /\right.$ $d T)_{T_{c}}$ on temperature $T$ in the localization region, $\sigma \ll \sigma^{*}$ (solid curve), and standard WHH dependence ${ }^{26}$ for dirty superconductors (dashed curve).

on $T$ observed for $\sigma \gg \sigma^{*}$ into a negative one in the region of $\sigma \leqslant \sigma^{*}$. Figure 4 shows $h_{c 2}(T)$ in the localization region, i.e., for $E_{\mathrm{F}}=E_{c}$.

Now we discuss the conditions when it is possible to neglect the magnetic field dependence of the diffusion coefficient $\tilde{D}\left(\omega_{m}\right)$. It is well known that a magnetic field diminishes the localization corrections due to the "maximally crossed diagrams" 27 and breaks the validity of Eq. (5). ${ }^{19}$ The relative change of $\tilde{D}\left(\omega_{m}\right)$ and the difference between $\phi$ and $\psi$ is apparently proportional to $\tilde{D}\left(\omega_{m}\right) H / \omega_{m} \phi_{0}$, and near $T_{c}$, when $\omega_{m} \sim T_{c}$, the change of $\tilde{D}\left(\omega_{m}\right)$ is small over the parameter $H \tilde{D}\left(T_{c}\right) / \phi_{0} T_{c}$. Thus, near $T_{c}$ we can neglect the influence of magnetic field $H_{c 2}$ on diffusion due to $\left|\left(T_{c}-T\right) / T_{c}\right| \ll 1$, and our method of calculation correctly determines the values of $k(a)$. However, possible magnetic field corrections to $r(a)$ may be important. For $T \rightarrow 0$ the critical field $H_{\mathrm{c} 2}$ grows, suppressing the localization corrections, and $\tilde{D}\left(\omega_{m}\right)$ grows, thus diminishing $H_{c 2}$. However, according to Coffey et al., ${ }^{13}$ this growth of $\tilde{D}\left(\omega_{m}\right)$ leads to the partial cancellation of the Coulomb contribution to the effective pairing constant $g$ (see below). This effect was studied by the authors of Ref. 13 for the metallic region and $\sigma>\sigma^{*}$. However, they did not take into account the $\omega$ dependence of the effective diffusion coefficient, which becomes important for $\sigma \sim \sigma^{*}$. Our results show that the appropriate changes of $H_{c 2}$ are not small, so that taking account of changes in $g$, as in Ref. 13, is not sufficient for the correct determination of $H_{c 2}(0)$. Thus, the final value of the correction to our estimate of $r(a)$ is not clear at present, and the difference between experimental values and our value of $r(a)$ can give an estimate of the magnetic field influence on the diffusion coefficient $\tilde{D}\left(\omega_{m}\right)$, i.e., upon localization.*

\footnotetext{
*Experimentally, dependences of $h_{c 2}(T)$ similar to that shown in Fig. 4 were observed by Tenhover $e t a l^{28}$ for amorphous MoRe. However their data for $k$ do not differ from the standard value 0.69 [in calculating $k$, they use the experimental values of $\sigma,\left(d H_{c 2} / d T\right)_{r_{c}}$, and of the coefficient $\gamma$ in the temperature dependence of the electronic specific heat].
} 


\section{COULOMB INTERACTION FOR STRONGLY DISORDERED SUPERCONDUCTORS}

\subsection{Coulomb Kernel for the Gap Equation}

In the BCS model discussed above we have assumed the existence of an effective pairing interaction $g$ in the energy region of the order of $2 \omega_{D}$ around the Fermi level. However, in real systems the pairing interaction is determined by the interplay between the attraction due to electron-phonon coupling and Coulomb repulsion. ${ }^{16}$ Clearly, for a strongly disordered system in the vicinity of the Anderson transition both interactions can change appreciably in comparison with a "pure" system.

It is well known that the Coulomb contribution to the effective pairing interaction is significantly weakened in comparison with the phonon contribution, due to the retarded nature of the electron-electron interaction via the exchange of virtual phonons. For the electron-phonon interaction the characteristic time is $\omega_{\mathrm{D}}^{-1}$, while for the Coulomb interaction in a "pure" system it is of the order of $E_{\mathrm{F}}^{-1}$ because this is the time during which the electrons pass each other in the Cooper pair. Both interactions are practically pointlike due to screening. With the growth of disorder an electron leaves the given region in space more slowly and this leads to an effective growth of Coulomb repulsion in the Cooper pair and to the corresponding lowering of $T_{c}$. This mechanism for the degradation of $T_{c}$ with disorder was studied by Anderson et al. ${ }^{12}$ using the scaling hypothesis. Below we shall consider this suppression of $T_{c}$ within the self-consistent theory. In the metallic region our estimates are in qualitative agreement with Ref. 12, although quantitatively they are different. However, our analysis of $T_{c}$ leads to the conclusion that superconductivity can survive in the localized phase if rather rigid conditions are satisfied.

In a strongly disordered system we must consider the matrix element of the screened Coulomb interaction $v\left(\mathbf{r}-\mathbf{r}^{\prime}\right)$ over exact eigenfunctions $\varphi_{\nu}(\mathbf{r})$ associated with exact eigenenergies $\varepsilon_{\nu}$ of an electron in the random field of this system:

$$
\left\langle\mu \nu\left|v\left(\mathbf{r}-\mathbf{r}^{\prime}\right)\right| \nu \mu\right\rangle=\int d \mathbf{r} \int d \mathbf{r}^{\prime} v\left(\mathbf{r}-\mathbf{r}^{\prime}\right) \varphi_{\mu}^{*}\left(\mathbf{r}^{\prime}\right) \varphi_{\nu}^{*}(\mathbf{r}) \varphi_{\mu}(\mathbf{r}) \varphi_{\nu}\left(\mathbf{r}^{\prime}\right)
$$

Averaging this matrix element over two isoenergetic surfaces $E_{\mathrm{F}}$ and $E_{\mathrm{F}}+\omega$ and over the disorder, we obtain the Coulomb kernel for the superconducting gap equation in the following form:

$$
\begin{aligned}
K_{\mathrm{C}}(\omega) & =\frac{1}{N\left(E_{\mathrm{F}}\right)}\left\langle\sum_{\mu \nu}\left\langle\mu \nu\left|v\left(\mathbf{r}-\mathbf{r}^{\prime}\right)\right| \nu \mu\right\rangle \delta\left(E_{\mathrm{F}}-\varepsilon_{\nu}\right) \delta\left(E_{\mathrm{F}}+\omega-\varepsilon_{\mu}\right)\right\rangle \\
& =\int d \mathbf{r} \int d \mathbf{r}^{\prime} v\left(\mathbf{r}-\mathbf{r}^{\prime}\right)\left\langle\left\langle\rho_{E_{\mathrm{F}}}(\mathbf{r}) \rho_{E_{\mathrm{F}}+\omega}\left(\mathbf{r}^{\prime}\right)\right\rangle\right.
\end{aligned}
$$


Here we have introduced Berezinskii-Gorkov spectral density ${ }^{29}$ :

$$
\begin{aligned}
\left\langle\rho_{E_{\mathrm{F}}}(\mathbf{r}) \rho_{E_{\mathrm{F}}+\omega}\left(\mathbf{r}^{\prime}\right)\right\rangle= & \frac{1}{N\left(E_{\mathrm{F}}\right)}\left\langle\sum_{\mu \nu} \varphi_{\nu}^{*}(\mathbf{r}) \varphi_{\mu}(\mathbf{r}) \varphi_{\mu}^{*}\left(\mathbf{r}^{\prime}\right) \varphi_{\nu}\left(\mathbf{r}^{\prime}\right)\right. \\
& \left.\times \delta\left(E_{\mathrm{F}}-\varepsilon_{\nu}\right) \delta\left(E_{\mathrm{F}}+\omega-\varepsilon_{\mu}\right)\right\rangle
\end{aligned}
$$

which gives the complete information on the nature of the electronic states. In particular, in the localization region, i.e., for $E_{\mathrm{F}}<E_{c}$, this spectral density contains a singular $\delta(\omega)$ contribution $^{29}$ :

$$
\left\langle\left\langle\rho_{E_{\mathrm{F}}}(\mathbf{r}) \rho_{E_{\mathrm{F}}+\omega}\left(\mathbf{r}^{\prime}\right)\right\rangle=\boldsymbol{P}\left(\mathbf{r}-\mathbf{r}^{\prime}\right) \delta(\omega)+\cdots\right.
$$

where

$$
P\left(\mathbf{r}-\mathbf{r}^{\prime}\right)=\frac{1}{N\left(E_{\mathrm{F}}\right)}\left\langle\sum_{\nu} \delta\left(E_{\mathrm{F}}-\varepsilon_{\nu}\right)\left|\varphi_{\nu}(\mathbf{r})\right|^{2}\left|\varphi_{\nu}\left(\mathbf{r}^{\prime}\right)\right|^{2}\right\rangle
$$

is the generalized inverse participation ratio connected with a finite probability of an electron returning to the initial point in an infinite time. ${ }^{30}$

Fourier transforming (27), we get

$$
K_{\mathrm{C}}(\omega)=\int \frac{d^{3} \mathbf{q}}{(2 \pi)^{3}} v(\mathbf{q})\left\langle\left\langle\rho_{E_{\mathrm{F}}} \rho_{E_{\mathrm{F}}+\omega}\right\rangle_{\mathbf{q}}\right.
$$

Below we shall assume a pointlike interaction $v(\mathbf{q})=v_{0}$. For $\omega \ll \tau^{-1}$ and $q \ll l^{-1}$ the Berezinskii-Gorkov spectral density posesses a diffusion contribution $^{30}$ :

$$
\left\langle\left\langle\rho_{E_{\mathrm{F}}} \rho_{E_{\mathrm{F}}+\omega}\right\rangle_{\mathbf{q}}^{\mathrm{d}}=\frac{1}{\pi N\left(E_{\mathrm{F}}\right)} \operatorname{Im} \phi^{\mathrm{RA}}(\mathbf{q} \omega)\right.
$$

Within the self-consistent theory of localization ${ }^{17-21} \phi^{\mathrm{RA}}(\mathbf{q} \omega)$ is determined by an analytic continuation $i \omega_{m} \rightarrow \omega+i \delta$ in (6). For the metallic region we have

$$
\phi^{\mathrm{RA}}(\mathbf{q} \omega)=-\frac{N\left(E_{\mathrm{F}}\right)}{\omega+i \tilde{D}(\omega) q^{2}}
$$

where

$$
\tilde{D}(\omega) \approx \begin{cases}D ; & |\omega| \ll \omega_{c}=\frac{1}{\tau}\left(\frac{\sigma}{\sigma_{0}}\right)^{3} \\ D_{0}(-i \omega \tau)^{1 / 3} ; & |\omega| \gg \omega_{c}\end{cases}
$$

Without disorder $\left(\tau^{-1}=0\right)$ the diffusion contribution vanishes and the kernel $K_{\mathrm{C}}(\omega)$ must reduce to the ordinary Coulomb potential $\mu=N\left(E_{\mathrm{F}}\right) v_{0}$. Thus 
we use the approximate relation

$$
\begin{aligned}
& K_{\mathrm{C}}(\omega) \approx \mu+K_{\mathrm{C}}^{\mathrm{d}}(\omega) \\
& K_{\mathrm{C}}^{\mathrm{d}}(\omega)=v_{0} \int \frac{d^{3} \mathbf{q}}{(2 \pi)^{3}}\left\langle\left\langle\rho_{E_{\mathrm{F}}} \rho_{E_{\mathrm{F}}+\omega}\right\rangle_{\mathrm{q}}^{\mathrm{d}}\right.
\end{aligned}
$$

reproducing the main difference between pure and disordered metals by the value $K_{\mathrm{C}}^{\mathrm{d}}(\omega)$. Using the above relations, we shall find $K_{\mathrm{C}}(\omega)$ and solve the linearized gap equation for the order parameter $\Delta(\omega)$ to determine $T_{c}$ and the conditions for the existence of superconductivity.

\subsection{Metallic Region}

From (32)-(34) we obtain*

$$
\begin{aligned}
K_{\mathrm{C}}^{\mathrm{d}}(\omega) & =v_{0} \int \frac{d^{3} \mathbf{q}}{(2 \pi)^{3}}\left\langle\left\langle\rho_{E_{\mathrm{F}}} \rho_{E_{\mathrm{F}}+\omega}\right\rangle\right\rangle_{\mathbf{q}}^{\mathrm{d}} \approx \frac{v_{0}}{2 \pi^{3}}\left\{\frac{1}{|\tilde{D}(\omega)| l}-\frac{|\omega|^{1 / 2}}{|\tilde{D}(\omega)|^{3 / 2}}\right\} \\
& \approx \frac{v_{0}}{2 \pi^{3}} \begin{cases}\frac{1}{D l}-\frac{|\omega|^{1 / 2}}{D^{3 / 2}} ; & |\omega| \ll \omega_{c} \\
\frac{1}{D_{0} l(\omega \tau)^{1 / 3}} ; & |\omega| \gg \omega_{c}\end{cases}
\end{aligned}
$$

Here we have introduced the upper outoff at $q$ of the order of $l^{-1} \cdot{ }^{31,32}$ Then we obtain the following approximate expression for the Coulomb kernel in the metallic region and near the mobility edge (see Figs. 5a and 5b):

$$
\begin{array}{rlrl}
K_{\mathrm{C}}(\omega)= & \mu \theta\left(E_{\mathrm{F}}-|\omega|\right) & \\
& +\frac{\mu}{k_{\mathrm{F}} l} \begin{cases}\frac{\sigma_{c}}{\sigma} ; & |\omega|<\omega_{c} \\
\frac{1}{k_{\mathrm{F}} l}(|\omega| \tau)^{-1 / 3} ; & \omega_{c}<|\omega|<\tau^{-1} \sim E_{\mathrm{F}}\end{cases}
\end{array}
$$

From (37) it can be readily seen that in the vicinity of the Anderson transition we obtain a considerable growth of the Coulomb repulsion due to diffusion renormalization, which was first considered by Altshuler and Aronov. ${ }^{32}$

The situation with regard to the electron-phonon contribution to the pairing interaction is different. Diffusion renormalization of the electronphonon vertex does not appear, ${ }^{33,34}$ because the appropriate corrections are

*These expressions actually define the Fock correction to the electronic density of states $-\delta N(E) / N\left(E_{\mathrm{F}}\right)$ due to electron-electron interaction..$^{31}$ In the region of "high" frequencies $|\omega| \gg \omega_{c}$ they slightly modify the expressions of Ref. 31 , where the $\omega$ dependence of $\tilde{D}(\omega) \sim$ $\omega^{1 / 3}$ was neglected. 

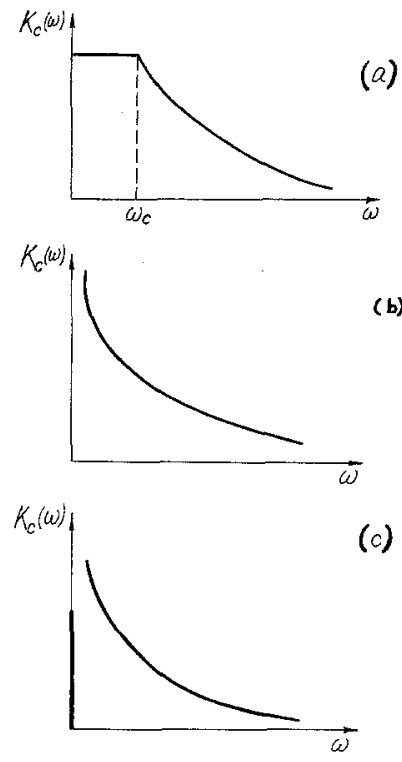

(c)

Fig. 5. Coulomb kernel (a) in the metallic region, (b) at the mobility edge, (c) and in the insulator.

cancelled when we take account of impurity vibrations. Of course, the value of the pairing interaction due to the electron-phonon interaction in a disordered system does change in comparison with the pure case. However, this change is relatively unimportant. ${ }^{12,33}$ Thus, following Ref. 12 , we can assume that this interaction is described by some dimensionless parameter $\lambda$, which is nonzero for the energies in an interval of the order of $2 \omega_{\mathrm{D}}$ around the Fermi level, and is weakly dependent on disorder.

The transition temperature $T_{c}$ is determined by the linearized gap equation, which we can take in the weak coupling form:

$$
\begin{aligned}
\Delta(\omega)= & \lambda \theta\left(\omega_{\mathrm{D}}-\omega\right) \int_{0}^{\omega_{\mathrm{D}}} \frac{d \omega^{\prime}}{\omega^{\prime}} \Delta\left(\omega^{\prime}\right) \operatorname{th} \frac{\omega^{\prime}}{2 T_{c}} \\
& -\theta\left(E_{\mathrm{F}}-\omega\right) \int_{0}^{E_{\mathrm{F}}} \frac{d \omega^{\prime}}{\omega^{\prime}} K_{\mathrm{C}}\left(\omega-\omega^{\prime}\right) \Delta\left(\omega^{\prime}\right) \text { th } \frac{\omega^{\prime}}{2 T_{c}}
\end{aligned}
$$

Consider first the metallic region and $\omega_{c} \gg \omega_{\mathrm{D}}$, which according to the estimate of $\omega_{c}$ in (34) corresponds roughly to $\sigma \geqslant \sigma_{c}$ for typical values of $E_{\mathrm{F}} / \omega_{\mathrm{D}} \approx 10^{2}$, i.e., the system is not very close to the Anderson transition. We calculate the change in $T_{c}$ due to a diffusion contribution in the Coulomb 
kernel (37), using perturbation th zory with respect to $K_{\mathrm{C}}^{\mathrm{d}}(\omega)$ :

$$
\begin{aligned}
\frac{\delta T_{c}}{T_{c 0}}= & \left\{\int_{0}^{\infty} \frac{d \omega}{\omega} \int_{0}^{\infty} \frac{d \omega^{\prime}}{\omega^{\prime}} \Delta_{0}(\omega) \text { th } \frac{\omega}{2 T_{c 0}} K_{\mathrm{C}}\left(\omega-\omega^{\prime}\right) \Delta_{0}\left(\omega^{\prime}\right) \text { th } \frac{\omega^{\prime}}{2 T_{c 0}}\right\} \\
& \times\left\{\frac{1}{2 T_{c 0}} \int_{0}^{\infty} d \omega\left[\Delta_{0}(\omega)\right]^{2}\left(\operatorname{ch} \frac{\omega}{2 T_{c 0}}\right)^{-2}\right\}^{-1}
\end{aligned}
$$

where $\Delta_{0}(\omega)$ is the zeroth-order solution (the usual two-step solution ${ }^{16}$ ) of (38) for the ordinary Coulomb kernel $K_{C}(\omega)=\mu \theta\left(E_{\mathrm{F}}-\omega\right)$. Using (37a), we obtain

$$
\frac{\delta T_{c}}{T_{c 0}} \approx-\frac{\mu}{\left(\lambda-\mu_{0}^{*}\right)^{2}} \frac{1}{k_{\mathrm{F}} l} \frac{\sigma_{c}}{\sigma}
$$

where

$$
T_{c 0}=1.13 \omega_{\mathrm{D}} \exp \left(-\frac{1}{\lambda-\mu_{0}^{*}}\right), \quad \mu_{0}^{*}=\frac{\mu}{1+\mu \ln \left(E_{\mathrm{F}} / \omega_{\mathrm{D}}\right)}
$$

are the usual expressions for the critical temperature of the pure system and the standard Coulomb pseudopotential. ${ }^{16}$ Actually the change of $T_{c}$ given by (40) is equivalent to the change of $\mu_{0}^{*}$ by the value

$$
\delta \mu^{*} \approx \mu \sigma_{c}^{2} / \sigma\left(\sigma+\sigma_{c}\right)
$$

where we have used (14) and $\sigma_{0} \approx \sigma_{c}\left(k_{\mathrm{F}} l\right)$ to exlude the factor of $\left(k_{\mathrm{F}} l\right)^{-1}$ in (40). According to (42), the Coulomb pseudopotential $\mu^{*}$ grows as $\sigma$ drops and this dependence on $\sigma$ here is stronger than in the similar expression of Ref. 12. This is due to our use of the expressions from the self-consistent theory of localization. The results of Ref. 12 can be obtained using another form of the generalized diffusion coefficient, equivalent to a scaling hypothesis on the $q$ dependence introduced by $\operatorname{Lee}^{35}: \tilde{D}(\omega \rightarrow 0, q) \approx$ $\left(D_{0} l\right) q$ for $q R_{l} \gg 1$. The self-consistent theory gives another limit: $\tilde{D}(\omega, q \rightarrow$ $0) \approx\left(D_{0} l\right)^{2 / 3}(-i \omega)^{1 / 3}$ at the mobility edge. Our expression for $\mu^{*}$ allows a noticeable change of $\mu^{*}$ for the conductivity region $\sigma \leqslant 10^{3} \Omega^{-1} \mathrm{~cm}^{-1}$. Such a dependence can explain the typical drop of $T_{c}$ in irradiated superconductors as their resistivity in the normal state grows ${ }^{24,25}$ in the Ioffe-Regel region. The expression for $\mu^{*}$ of Ref. 12 can explain the experimental data only by assuming that the values of the conductivity scale an order of magnitude larger than the typical Ioffe-Regel value, for which there seems to be no valid theoretical foundation.

Consider now the situation at the mobility edge itself, when $\sigma=0$ and $\omega_{c}=0$, and $K_{C}(\omega)$ is determined by the second expression in (37) for all frequencies below $\tau^{-1} \sim E_{\mathrm{F}}$ (see Fig. 5b). In this case, as is shown in 
Appendix B, the Coulomb effect on $T_{c}$ can again be represented by the effective Coulomb pseudopotential $\mu^{*}$. However, now we have ( $\alpha=$ const $\approx 1$ )

$$
\mu^{*} \approx \alpha \mu\left(\omega_{\mathrm{D}} \tau\right)^{-1 / 3}
$$

The value of $T_{c}$ can remain finite at the mobility edge under rather rigid conditions: the parameters $E_{\mathrm{F}} \sim \tau^{-1}$ and $\mu$ must be sufficiently small, while $\lambda$ must be close to unity. As a crude estimate we take $\lambda \approx 1, \mu \leqslant 0.2$, and $E_{\mathrm{F}} \leqslant 10^{3} T_{c 0}$. Apparently such a situation can be realized in some Chevrel phase superconductors ${ }^{36}$ (see below).

Using (42) and (43), we can write down a simple interpolation formula for the dependence of $\mu^{*}$ on $\sigma$ :

$$
\begin{gathered}
\mu^{*}(\sigma)=\mu_{0}^{*}+\frac{\alpha \mu\left(\omega_{\mathrm{D}} \tau\right)^{-1 / 3}-\mu_{0}^{*}}{1+\left(\omega_{\mathrm{D}} \tau\right)^{-1 / 3} \sigma\left(\sigma+\sigma_{c}\right) / \sigma_{c}^{2}} \\
\omega_{\mathrm{D}} \tau=\frac{\omega_{\mathrm{D}}}{2 E_{\mathrm{F}}}\left(1+\frac{\sigma}{\sigma_{c}}\right)
\end{gathered}
$$

This expression describes the smooth crossover from the region where there is a weak effect of localization on $T_{c}$ [Eq. (42)] to the vicinity of the Anderson transition [Eq. (43)] at $\omega_{c} \approx \omega_{\mathrm{D}}$.

In Fig. 6 we compare the theoretical predictions of Eq. (44) with the experimental data for $T_{c}$ obtained in Ref. 24 for $\mathrm{SnMo}_{5} \mathrm{~S}_{6}$. We have calculated the dependence of $T_{c}$ on $\sigma$ using the standard McMillan formula for $T_{c}{ }^{12}$ with $\mu^{*}(\sigma)$ given by (44). Following Ref. 24 , we take the preexponential factor in the McMillan formula to be equal to $125 \mathrm{~K}, \mu_{0}^{*}=0.1$, and $\lambda=1.06$. Then, for $E_{\mathrm{F}} / \omega_{\mathrm{D}} \approx 5$, we get $\mu \approx 0.13$. The theoretical curves in Fig. 6 are given for $\sigma_{c} \approx 1500 \Omega^{-1} \mathrm{~cm}^{-1}$ and $\alpha \approx 1.5$ and 2.0 in (44). Taking into account the crudeness of our theory, the agreement is quite satisfactory. Further discussion will be given in Section 4 .

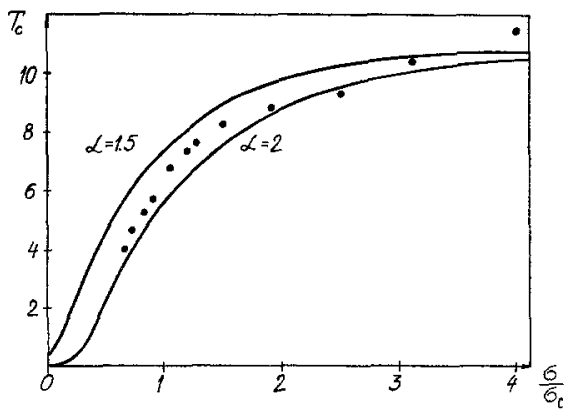

Fig. 6. Transition temperature versus conductivity. Comparison of theoretical curves with experimental data of Ref. 24. See text for details. 


\subsection{Localization Region}

Consider now the region of $E_{\mathrm{F}}<E_{c}$, i.e., an Anderson insulator. According to (27) and (29), in this case the Coulomb kernel has a $\delta(\omega)$ contribution connected to electron repulsion in one quantum state [see (30) and Ref. 31 ; see also Fig. 5c]:

$$
K_{\mathrm{C}}^{1}(\omega)=v_{0} P \delta(\omega) ; \quad P=\left.P\left(\mathbf{r}-\mathbf{r}^{\prime}\right)\right|_{\mathrm{r}=\mathbf{r}^{\prime}} \sim R_{l}^{-3}
$$

This mechanism acts in addition to those considered above. Using (45) as a full Coulomb kernel, we can solve (38) exactly (Appendix B). Then we obtain an equation for $T_{c}$ in the approximate form

$$
\ln \frac{T^{*}}{T_{\mathrm{c}}} \approx \psi\left(\frac{1}{2}+\frac{\mu P}{4 T_{\mathrm{c}} N\left(E_{\mathrm{F}}\right)}\right)-\psi\left(\frac{1}{2}\right)
$$

where $T^{*}$ is taken as the critical temperature at the mobility edge, i.e., determined by (41) with $\mu_{0}^{*}$ replaced by (43). In this way we actually overestimate the influence of Coulomb repulsion in the localization region. We see that this extra repulsion acts upon the superconducting $T_{c}$ as magnetic impurities ${ }^{16}$ with an effective spin-flip time:

$$
1 / \tau_{\mathrm{sf}}=\pi \mu P / N\left(E_{\mathrm{F}}\right) \sim \mu / N\left(E_{\mathrm{F}}\right) R_{l}^{3}
$$

Superconductivity survives for $\tau_{\mathrm{sf}}^{-1}<0.57 T^{*}$, i.e., for

$$
R_{l}>\left[\mu / N\left(E_{\mathrm{F}}\right) T_{c}^{*}\right]^{1 / 3} \sim\left(\xi_{0} k_{\mathrm{F}}^{-2}\right)^{1 / 3} \sim\left(\xi_{0} l^{2}\right)^{1 / 3}
$$

where the last estimates are roughly valid for typical parameters and correspond to the condition (18). Thus the Coulomb repulsion of electrons in the one-quantum state, important in the localization region, ${ }^{31}$ leads to a rapid destruction of superconductivity. The size of a possible coexistence region is roughly determined by (18) and (48).

The Coulomb gap effects ${ }^{37}$ are unimportant here. The width of the Coulomb gap, according to Efros and Shklovskii, ${ }^{37}$ is given by the estimate

$$
\Delta_{\mathrm{C}} \approx\left(e^{2} / \kappa^{3 / 2}\right)\left[N\left(E_{\mathrm{F}}\right)\right]^{1 / 2}
$$

where $\kappa$ is the dielectric function in the insulator region. Near the mobility edge, in the self-consistent theory we have ${ }^{31}$

$$
\kappa \approx 4 \pi e^{2} N\left(E_{\mathrm{F}}\right) R_{l}^{2}
$$

Thus $\Delta_{\mathrm{C}} \sim\left[N\left(E_{\mathrm{F}}\right) R_{l}^{3}\right]^{-1}$ and $\Delta_{\mathrm{C}} \ll T_{\mathrm{c}}$ if the condition (18) is satisfied. So the Coulomb gap can be safely neglected in the "coexistence" region.

This treatment again assumes that the electron-electron interaction is weak $(\mu \ll 1)$ and can be described by the lowest order of perturbation theory. The influence of this interaction upon the metal-insulator transition, 
i.e., on the spectral density (28), is neglected. ${ }^{31}$ However, one has to keep in mind the possible importance of Coulomb interactions in a real system, although, as we have mentioned, a complete theory of the metal-insulator transition in disordered systems is still lacking.

\section{CONCLUSION}

An experimental investigation of the effects discussed in this paper seems possible by the study of "high-temperature" superconductors disordered by irradiation with fast neutrons. Among the numerous experiments of this kind, the most interesting appear to be some results on irradiated molybdenum sulfides (Chevrel phase superconductors). For these compounds high values of initial $T_{c 0} \approx 15 \mathrm{~K}$ are typical, as well as rather narrow energy bands. According to band-structure calculations, ${ }^{36}$ the Fermi level in these compounds is very close to the upper edge of the conduction band, and characteristic values of $E_{\mathrm{F}}$ are of the order of $10^{3} \mathrm{~K}$. These values seem to be ideal from the point of view of the above criteria.

We remark upon the results of studies of irradiated $\mathrm{SnMo}_{5} \mathrm{~S}_{6}$ (Ref. 24) and $\mathrm{Pb}_{1-x} \mathrm{U}_{x} \mathrm{Mo}_{6} \mathrm{~S}_{8}{ }^{25}$ Strong disordering of these compounds leads to a lowering of $T_{c}$ to values of the order of $1 \mathrm{~K}$, with the corresponding growth of the residual resistance up to values of several units of $10^{-3} \Omega-\mathrm{cm}$, in agreement with the estimates of minimal metallic conductivity due to Mott and Davis. ${ }^{23}$ The temperature coefficient of resistance becomes negative at all temperatures and of quite a significant value. The observed resistance is greater than the values typical for most "dirty" alloys from the IoffeRegal-Mooij region. ${ }^{38}$ From the point of view of empirical criteria for localization, ${ }^{23}$ these results seem very attractive. We have already noted that the investigated behavior of $\left(d H_{c 2} / d T\right)_{T_{\mathrm{c}}}$ in these systems is also in qualitative agreement with theoretical predictions.

Interestingly, the situation with regard to $\sigma_{c}$ also seems satisfying (see Fig. 6). The value of $\sigma_{c} \approx 10^{3} \Omega^{-1} \mathrm{~cm}^{-1}$, although different from the estimates of minimal metallic conductivity, are more appropriate than the values of this parameter determined from similar fits in Ref. 12. It has already been noted ${ }^{39}$ that the "critical region" in $\sigma$ during the metal-insulator transition in impurity bands is rather large experimentally. Accordingly, the values of $\sigma_{c}$ determined from these experiments are an order of magnitude larger than the Mott estimates for $\sigma_{c}$. This seems to be in accordance with our values of $\sigma_{c}$ determined from the $T_{c}$ dependence on $\sigma$. In any event, one should not have expected good agreement between such a crude theory and experiment, and these data allow us to claim with some confidence that these compounds, irradiated with a sufficiently large fluence of fast neutrons, are really in the vicinity of the Anderson transition, while conserving 
superconductivity. Of course, on the basis of existing data we cannot claim that either of these systems is actually in the state of an Anderson insulator. In these respects the accurate measurement of resistivity for very low temperatures in the normal state [i.e., for external magnetic fields greater than $\left.H_{c 2}(0)\right]$ may be very important.

Finally, we note that the strong anisotropy of electron motion and relatively narrow energy bands in recently discovered organic superconductors $^{40}$ can lead to the possibility of Anderson localization in these systems for a weak disorder, i.e., for $\tau^{-1} \ll E_{\mathrm{F}}$, so that the criteria for the coexistence of superconductivity and localization may greatly improve.

\section{APPENDIX A}

Here we give some details of the derivation of the expressions (15) and (16) for the GL coefficients. Using (3), (5), and (6), we obtain

$$
A=\frac{1}{g}-2 N\left(E_{\mathrm{F}}\right) \sum_{n \geqslant 0}^{n^{*}} \frac{1}{2 n+1}=\frac{1}{g}-N\left(E_{\mathrm{F}}\right) \ln 1.13 \frac{\omega_{\mathrm{D}}}{T}=N\left(E_{\mathrm{F}}\right) \ln \frac{T}{T_{\mathrm{c}}}
$$

where $n^{*}=\omega_{\mathrm{D}} / 2 \pi T$ has been introduced to cut off the logarithmic divergence, taking into account that electron attraction exists in the energy region of $2 \omega_{\mathrm{D}}$ around the Fermi level. The generalized diffusion coefficient $\tilde{D}\left(\omega_{m}\right)$ does not contribute [due to $q=0$ in (3)]. This is a reflection of the Anderson theorem ${ }^{14}$ : disorder influences $T_{c}$ only through changes in the density of states $N\left(E_{\mathrm{F}}\right)$.

We shall calculate the coefficient $B$, neglecting the weak dependence on q. Then it is seen from Fig. 7 that the contribution of the diagrams in Figs. 7a and $7 \mathrm{~b}$ is small in comparison with that of Fig. 7a. The "triangular" vertex can be found in the self-consistent theory of localization as described in Ref. 31. We have

$$
\gamma\left(\mathbf{q}=0, \omega_{m}=2 \varepsilon_{n}\right) \approx 1+1 /\left(2 \tau\left|\varepsilon_{n}\right|\right)
$$

where the first term takes account of "high" frequencies, while the next is

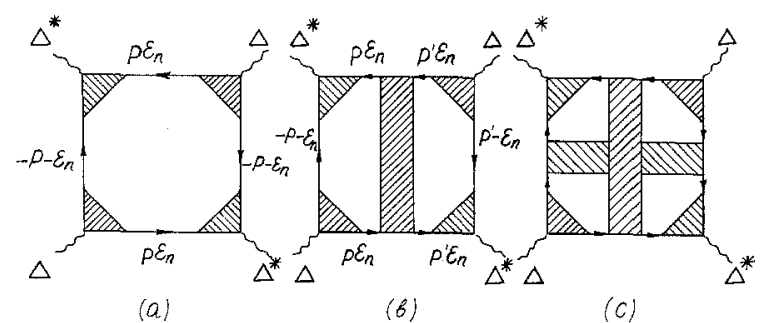

Fig. 7. Diagrams for calculating the coefficient $B$. 
a diffusion contribution. Then, from Fig. 7a we get

$$
\begin{aligned}
B & \approx N\left(E_{\mathrm{F}}\right) T \sum_{\varepsilon_{n}} \int_{-\infty}^{\infty} d \xi_{\mathrm{p}} \gamma^{4}\left(\mathbf{q}=0, \omega_{m}=2 \varepsilon_{n}\right) G^{2}\left(\varepsilon_{n} \xi_{\mathrm{p}}\right) G^{2}\left(-\varepsilon_{n} \xi_{\mathrm{p}}\right) \\
& =N\left(E_{\mathrm{F}}\right) T \frac{\pi}{2} \sum_{n \geqslant 0} \frac{1}{\varepsilon_{n}^{3}}=\frac{7 \zeta(3)}{8 \pi^{2} T} N\left(E_{\mathrm{F}}\right)
\end{aligned}
$$

where

$$
G\left(\varepsilon_{n} \xi_{\rho}\right)=\left(i \varepsilon_{n}-\xi_{\rho}+\frac{i}{2 \tau} \frac{\varepsilon_{n}}{\left|\varepsilon_{n}\right|}\right)^{-1}
$$

is the usual approximation for the one-particle Green's function used in the self-consistent theory. ${ }^{18-21}$ Consider now calculations for $C$. Using (4)-(6), we find for the metallic region $\left(E_{\mathrm{F}}>E_{c}\right)$, not very close to the mobility edge, when $\tilde{D}\left(\omega_{m}\right)=D$,

$$
\begin{aligned}
C & =-\left.i \pi T N\left(E_{\mathrm{F}}\right) \sum_{\varepsilon_{n}} \frac{\partial^{2}}{\partial q^{2}} \frac{1}{2 i\left|\varepsilon_{n}\right|+i D q^{2}}\right|_{q=0} \\
& =\pi T N\left(E_{\mathrm{F}}\right) D \sum_{\varepsilon_{n}} \frac{1}{2 \varepsilon_{n}^{2}}=\frac{N\left(E_{\mathrm{F}}\right) D}{\pi T} \sum_{n \geqslant 0} \frac{1}{(2 n+1)^{2}}=\frac{\pi}{8 T} N\left(E_{\mathrm{F}}\right) D
\end{aligned}
$$

Analogously, for an insulator $\left(E_{\mathrm{F}}<E_{c}\right)$, but also not so close to the mobility edge, when

$$
\tilde{D}\left(\omega_{m}\right)=D \frac{\omega_{m}}{\omega_{m}+3 D \omega_{0}^{2} / v_{\mathrm{F}}^{2}}
$$

[see (8)], we obtain

$$
\begin{aligned}
C & =\frac{\pi}{2} T N\left(E_{\mathrm{F}}\right) \sum_{\varepsilon_{n}} \frac{1}{\varepsilon_{n}^{2}} \tilde{D}\left(2\left|\varepsilon_{n}\right|\right) \\
& =\pi T N\left(E_{\mathrm{F}}\right) D \sum_{\varepsilon_{n}} \frac{1}{(2 n+1)^{2}+(2 n+1) 3 D \omega_{0}^{2} / 2 \pi T v_{\mathrm{F}}^{2}} \\
& =\frac{N\left(E_{\mathrm{F}}\right) v_{\mathrm{F}}^{2}}{3 \omega_{0}^{2}}\left[\psi\left(\frac{1}{2}+\frac{3 D \omega_{0}^{2}}{4 \pi T v_{\mathrm{F}}^{2}}\right)-\psi\left(\frac{1}{2}\right)\right] \\
& =N\left(E_{\mathrm{F}}\right) R_{l}^{2}\left[\psi\left(\frac{1}{2}+\frac{D}{4 \pi T R_{l}^{2}}\right)-\psi\left(\frac{1}{2}\right)\right] \approx N\left(E_{\mathrm{F}}\right) R_{l}^{2} \ln \frac{1.78 D}{\pi T R_{l}^{2}}
\end{aligned}
$$

where the approximate expression is valid for $D R_{l}^{-2} \gg 4 \pi T$.

In the vicinity of the mobility edge, both for a metal and an insulator, we have $\mathrm{e}^{18,21}$

$$
\tilde{D}\left(\omega_{m}\right) \approx(\pi / 2)^{2 / 3} D_{0}\left(\omega_{m} \tau\right)^{1 / 3}=(\pi / 2 \sqrt{3})^{2 / 3}\left(D_{0} l\right)^{2 / 3} \omega_{m}^{1 / 3}
$$


so that

$$
\begin{aligned}
C & =\frac{\pi}{2} T N\left(E_{\mathrm{F}}\right) \sum_{\varepsilon_{n}} \frac{1}{\varepsilon_{n}^{2}} D\left(2\left|\varepsilon_{n}\right|\right)=\frac{\pi}{2}\left(\frac{\pi}{\sqrt{6}}\right)^{2 / 3}\left(D_{0} l\right)^{2 / 3} T N\left(E_{\mathrm{F}}\right) \sum_{\varepsilon_{n}} \frac{1}{\left|\varepsilon_{n}\right|^{5 / 3}} \\
& =\frac{1}{6^{1 / 3}}\left(\frac{D_{0} l}{T}\right)^{2 / 3} N\left(E_{\mathrm{F}}\right) \sum_{n \geqslant 0} \frac{1}{(2 n+1)^{5 / 3}} \\
& =\frac{1}{6^{1 / 3}}\left(1-\frac{1}{2^{5 / 3}}\right) \zeta\left(\frac{5}{3}\right) N\left(E_{\mathrm{F}}\right)\left(\frac{D_{0} l}{T}\right)^{2 / 3}
\end{aligned}
$$

Expression (A7) dominates over (A5) for

$$
D / T_{c} \approx D_{0} l / R_{l} T_{c} \leqslant D_{0}^{2 / 3}\left(l / T_{c}\right)^{2 / 3}
$$

which defines the limits of applicability given in (16).

\section{APPENDIX B}

At the mobility edge $\omega_{c}=0$ and the Coulomb kernel in (38) is determined mainly by the second term in (37b). Here we try to find a solution of (38) in the form

$$
\Delta(\omega)=\Delta_{1} \theta\left(\omega_{D}-|\omega|\right)+f(\omega)
$$

Then we get an integral equation for $f(\omega)$,

$$
f(x)=\tilde{\mu} \Delta_{1} F(x)-\tilde{\mu} \int_{0}^{E_{\mathrm{F}} / \omega_{\mathrm{D}}} d x^{\prime} g\left(x-x^{\prime}\right) f\left(x^{\prime}\right) \frac{1}{x^{\prime}} \operatorname{th} \frac{\omega_{\mathrm{D}}}{2 T_{c}} x^{\prime}
$$

where

$$
\begin{aligned}
& F(x)=\int_{0}^{1} d x^{\prime} g\left(x-x^{\prime}\right) \frac{1}{x^{\prime}} \operatorname{th} \frac{\omega_{D}}{2 T_{c}} x^{\prime} \\
& g(x)=x^{-1 / 3} ; \quad x \approx \omega / \omega_{D} ; \quad \tilde{\mu}=\mu\left(\omega_{D} \tau\right)^{-1 / 3}
\end{aligned}
$$

Here $F(x) / \ln \left(\omega_{\mathrm{D}} / T_{c}\right)$ changes from 4.2 to 1 for $x$ changing from 0 to 1 , and for $x \gg 1$ we get the asymptotic behavior $F(x)=x^{-1 / 3} \ln \left(\omega_{D} / T_{c}\right)$. Using the small difference between $\left(\omega_{D} / T_{c}\right)^{1 / 3}$ and $\ln \left(\omega_{D} / T_{c}\right)$ for any reasonable values of $\omega_{\mathrm{D}}$ and $T_{c}$ and the weak dependence of $F(x)$ on $x$ for $0<x<1$, we come to the conclusion that the unknown function $f(x)$ from (B2) is weakly $x$ dependent for $x<1$ and $f(x)-x^{-1 / 3}$ for $x>1$. Taking all this into account, we get the following equation for $T_{c}$ :

$$
1=\lambda \ln \frac{\omega_{\mathrm{D}}}{T_{c}}\left[1-\frac{\left(\beta_{1} \tilde{\mu}-m\right) \ln \left(\omega_{\mathrm{D}} / T_{c}\right)}{1+\left(\beta_{2} \tilde{\mu}-m\right) \ln \left(\omega_{\mathrm{D}} / T_{c}\right)}\right]
$$


where

$$
\begin{aligned}
& \beta_{1}=\left(\ln \frac{\omega_{\mathrm{D}}}{T_{c}}\right)^{-2} \int_{0}^{1} d x \int_{0}^{1} d x^{\prime} \frac{1}{x x^{\prime}\left|x-x^{\prime}\right|^{1 / 3}} \operatorname{th} \frac{\omega_{\mathrm{D}}}{2 T_{c}} x \operatorname{th} \frac{\omega_{\mathrm{D}}}{2 T_{c}} x^{\prime} \approx 1 \\
& m=\frac{3}{2} \tilde{\mu}^{2} /\left(1+\beta_{3} \tilde{\mu}\right) ; \quad 1<\beta_{2}<4.2 ; \quad \frac{3}{2}<\beta_{3}<3
\end{aligned}
$$

For $\tilde{\mu} \gg 1$ we have $\beta_{2} \approx 1$ and $\beta_{3} \approx 3$. Thus from (B4) we obtain an estimate $\mu^{*} \approx \beta \tilde{\mu}$ with $0.5<\beta<3$; actually $\beta \approx 1$ for large values of $\tilde{\mu}$. This is the result given as (43).

Below the mobility edge $\left(E_{\mathrm{F}}<E_{c}\right)$ we can solve (38) with the Coulomb kernel given in (45) exactly. The additional Coulomb effects considered above, connected with "regular" contributions to the spectral density (28), ${ }^{31}$ can be taken into account with a simple substitution $\lambda \rightarrow \lambda^{*}=\lambda-\mu^{*}$, where $\mu^{*}$ is given by (43). Actually, we can convince ourselves that such a procedure overestimates the Coulomb repulsion in the localization region. It is easily seen that the solution of (38) in this case can be written as

$$
\Delta(\omega)=\frac{\theta\left(\omega_{\mathrm{D}}-|\omega|\right) \Delta_{1}}{1+\left[\mu P / 2 N\left(E_{\mathrm{F}}\right) \omega\right] \operatorname{th}\left(\omega / 2 T_{c}\right)}
$$

where

$$
\Delta_{1}=\lambda^{*} \int_{0}^{\omega_{\mathrm{D}}} d \omega \Delta(\omega) \frac{1}{\omega} \operatorname{th} \frac{\omega}{2 T_{c}}
$$

giving the equation for $T_{c}$ of the following form:

$$
1=\lambda^{*} \int_{0}^{\omega_{\mathrm{D}}} d \omega \frac{\operatorname{th}\left(\omega / 2 T_{c}\right)}{\omega+\left[\mu P / 2 N\left(E_{\mathrm{F}}\right)\right] \operatorname{th}\left(\omega / 2 T_{c}\right)}
$$

which reduces to (46) with rather good accuracy.

\section{ACKNOWLEDGMENTS}

The authors appreciate discussions on different theoretical aspects of the problem with O. V. Dolgov, E. G. Maksimov, V. N. Fleurov, D. E. Khmelnitskii, and D. I. Khomskii, as well as information on experimental investigations of irradiated superconductors and discussions with N. E. Alekseevskii, V. E. Arkhipov, and B. N. Goschitskii. They thank Prof. V. L. Ginzburg for his interest in this work. One of the authors (MVS) is grateful for the hospitality extended to him during his stay at the I. E. Tamm Department of Theoretical Physics of the P. N. Lebedev Physical Institute, where this work was performed. 


\section{REFERENCES}

1. M. V. Sadovskii, Usp. Fiz. Nauk 133, 223 (1981) [Sov. Phys. Uspekhi 24, 96 (1981)].

2. Y. Nagaoka and H. Fukuyama, eds., Anderson Localization (Springer, Berlin, 1982).

3. P. W. Anderson, Phys. Rev. 189, 1492 (1958).

4. J. Bardeen, L. N. Cooper, and J. R. Schrieffer, Phys. Rev. 106, 162 (1957).

5. Y. Imry and M. Strongin, Phys. Rev. B 24, 6353 (1981).

6. S. Maekawa and H. Fukuyama, J. Phys. Soc. Japan 51, 1380 (1981).

7. S. Maekawa, in Anderson Localization, Y. Nagaoka and H. Fukuyama, eds. (Springer, Berlin, 1982), p. 103.

8. H. Takagi and Y. Kuroda, Solid State Commun. 41, 643 (1982).

9. H. Takagi, R. Souda, and Y. Kuroda, Prog. Theor. Phys. 68, 426 (1982).

10. H. Takagi and Y. Kuroda, Prog. Theor. Phys. 69, 1677 (1983).

11. R. Oppermann, J. Phys. Soc. Japan 52, 3554 (1983).

12. P. W. Anderson, K. A. Muttalib, and T. V. Ramakrishnan, Phys. Rev. B 28, 117 (1983).

13. L. Coffey, K. A. Muttalib, and K. Levin, Phys. Rev. Lett. 52, 783 (1984).

14. P. W. Anderson, J. Phys. Chem. Solids 11, 26 (1959).

15. L. P. Gorkov, Zh. Eksp. Teor. Fiz. 37, 1407 (1959).

16. P. G. de Gennes, Superconductivity of Metals and Alloys (W. A. Benjamin, New York, 1966).

17. W. Götze, J. Phys. C 12, 1279 (1979); Philos. Mag. 43, 219 (1981).

18. D. Vollhardt and P. Wölfle, Phys. Rev. B 22, 4666 (1980); Phys. Rev. Lett. 48, 699 (1982); in Anderson Localizations, Y. Nagaoka and H. Fukuyama, eds. (Springer, Berlin, 1982), p. 26.

19. D. Yoshioka, Y. Ono, and H. Fukuyama, J. Phys. Soc. Japan 50, 3419 (1981).

20. A. V. Myasnikov and M. V. Sadovskii, Fiz. Tverd. Tela 24, 3569 (1982).

21. B. Shapiro, Phys. Rev. B 25, 4266 (1982).

22. A. F. Ioffe and A. R. Regel, Prog. Semicond. 4, 237 (1960).

23. N. F. Mott and E. A. Davis, Electron Processes in Non-Crystalline Materials (Clarendon Press, Oxford, 1979).

24. S. A. Davydov, V. E. Arkhipov, V. I. Voronin, and B. N. Goschitskii, Fiz. Metallov. Metalloved. 55, 931 (1983).

25. N. E. Alekseevskii, A. V. Mitin, V. N. Samosyuck, and V. I. Firsov, Zh. Eksp. Teor. Fiz. 85, 1092 (1983).

26. N. R. Wethamer, E. Helfand, and P. C. Hohenberg, Phys. Rev. 147, 295 (1966).

27. B. L. Altshuler, D. E. Khmelnitskii, A. I. Larkin, and P. A. Lee, Phys. Rev. B 22, 5142 (1980).

28. M. Tenhover, W. L. Johnson, and C. C. Tsuei, Solid State Commun. 38, 53 (1981).

29. V. L. Berezinskii and L. P. Gorkov, Zh. Eksp. Teor. Fiz. 77, 2498 (1979).

30. M. V. Sadovskii, Zh. Eksp. Teor. Fiz. 83, 1418 (1982).

31. M. I. Katsnelson and M. V. Sadovskii, Fiz. Tverd. Tela 25, 3372 (1983); Zh. Eksp. Teor. Fiz. 87, 523 (1984).

32. B. L. Altshuler and A. G. Aronov, Zh. Eksp. Teor. Fiz. 77, 2028 (1979).

33. B. Keck and A. Schmid, J. Low Temp. Phys. 24, 611 (1976).

34. V. N. Fleurov, P. S. Kondratenko, and A. N. Kozlov, J. Phys. F 10, 1953 (1980).

35. P. A. Lee, Phys. Rev. B 26, 5882 (1982).

36. A. J. Freeman and T. Jarlborg, in Superconductivity in Ternary Compounds II. Superconductivity and Magnetism, M. B. Maple and F. Fischer, eds. (Springer Verlag, Berlin, 1982), p. 167.

37. A. L. Efros and B. I. Shklovskii, J. Phys. C 8, L49 (1975).

38. J. H. Mooij, Phys. Stat. Sol. (a) 17, 521 (1973).

39. T. F. Rosenbaum, K. Andres, G. A. Thomas and R. N. Bhatt, Phys. Rev. Lett. 45, 1723 (1980); G. A. Thomas, M. A. Paalanen, and T. F. Rosenbaum, Phys. Rev. B 27, 3897 (1983).

40. D. Jerome and H. J. Schulz, Adv. Phys. 31, 299 (1982); J. Friedel and D. Jerome, Contemp. Phys. 23, 583 (1982). 